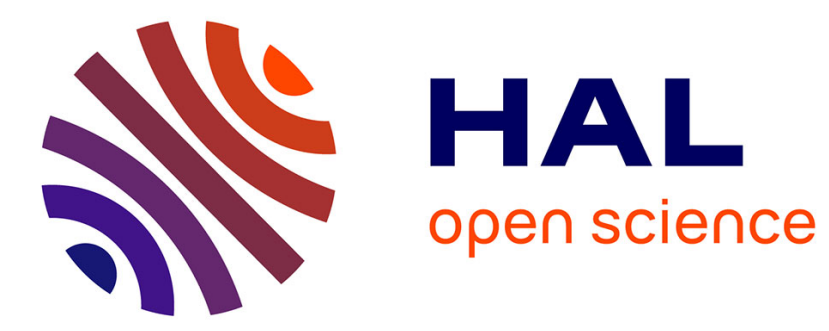

\title{
ON THE LOJASIEWICZ EXPONENTS OF QUASI-HOMOGENEOUS FUNCTIONS
}

\author{
Alain Haraux, Tien Son Pham
}

\section{To cite this version:}

Alain Haraux, Tien Son Pham. ON THE LOJASIEWICZ EXPONENTS OF QUASIHOMOGENEOUS FUNCTIONS. Journal of Singularities, 2015, 11, pp.52-66 10.5427/jsing.2015.11c . hal-01246013

\section{HAL Id: hal-01246013 https://hal.sorbonne-universite.fr/hal-01246013}

Submitted on 18 Dec 2015

HAL is a multi-disciplinary open access archive for the deposit and dissemination of scientific research documents, whether they are published or not. The documents may come from teaching and research institutions in France or abroad, or from public or private research centers.
L'archive ouverte pluridisciplinaire HAL, est destinée au dépôt et à la diffusion de documents scientifiques de niveau recherche, publiés ou non, émanant des établissements d'enseignement et de recherche français ou étrangers, des laboratoires publics ou privés. 


\title{
ON THE LOJASIEWICZ EXPONENTS OF QUASI-HOMOGENEOUS FUNCTIONS
}

\author{
Alain HARAUX \\ Laboratoire Jacques-Louis Lions, U.M.R C.N.R.S. 7598, \\ Université Pierre et Marie Curie, \\ Bô̂te courrier 187, 75252 Paris Cedex 05, France \\ haraux@ann.jussieu.fr \\ Tien Son PHẠM ${ }^{1}$ \\ Department of Mathematics, Dalat University, Dalat, Vietnam \\ sonpt@dlu.edu.vn
}

Résumé. Les fonctions quasi-homogènes, en particulier les polynômes, possèdent des propriétés particulières au voisinage de l'origine qui permettent d'estimer les exposants dits de Łojasiewicz d'une manière tout à fait analogue à ceux des fonctions homogènes. En particulier on généralise un résultat antérieur pour les polynômes à deux variables concernant l'iné galité du gradient de Łojasiewicz optimale á l'origine.

\begin{abstract}
Quasi-homogeneous functions, and especially polynomials, enjoy some specific properties around the origin which allow to estimate the so-called Lojasiewicz exponents in a way quite similar to homogeneous functions. In particular we generalize a previous result for polynomials of two variables concerning the optimal Łojasiewicz gradient inequality at the origin.
\end{abstract}

AMS classification numbers:14P10, 14P15, 32S05, 34A26

Keywords: quasi-homogeneous, gradient inequality, Łojasiewicz exponents.

\footnotetext{
${ }^{1}$ The second author was partially supported by Vietnam National Foundation for Science and Technology Development (NAFOSTED) grant number 101.04-2013.07
} 


\section{INTRODUCTION}

In his pioneering papers [13], [14], Łojasiewicz established that any analytic function $f$ of $n$ real variables satisfies an inequality of the form

$$
\|\nabla f(x)\| \geq c|f(x)-f(a)|^{\beta}
$$

for $\|x-a\|$ small enough with $c>0, \beta \in(0,1)$. This inequality, known as the Łojasiewicz gradient inequality, is useful to establish trend to equilibrium of the general solutions of gradient systems

$$
u^{\prime}+\nabla f(u)=0
$$

and can also be used to evaluate the rate of convergence. It is therefore of interest to know as precisely as possible the connection between $f$ and its gradient and in particular to determine the best(smallest) possible value of $\beta$ in (1.1) when $a$ is a critical point of $f$. This value is called the Eojasiewicz exponent at $a$. In [7] for instance, it was shown that if $f$ is a homogeneous polynomial with degree $d \geq 2$, the Łojasiewicz exponent at the origin is exactly $1-\frac{1}{d}$ when $n=2$. This property is no longer true if $n>2$.

On the other hand, Gwoździewicz [6] (see also [16]) considered the case of a real analytic function at an isolated zero and also found, in this case, an interesting relationship between various Łojasiewicz exponents, relative to different Łojasiewicz inequalities. In addition the case of general polynomials has been thoroughly investigated by D'Acunto and Kurdyka in [2] (see also [11, 12, 17]).

Our paper is concerned to the extension of the result from [7] and several estimates of Łojasiewicz exponents at the origin when $f$ is a quasi-homogeneous map (see, for example, [1]). It is divided in 5 sections. In Section 2, we state and prove some preliminary results, mainly concerning the local behavior of quasi-homogeneous maps near the origin. Section 3 contains more information in the specific case where the origin is an isolated zero of $f$. Section 4 deals with the Eojasiewicz gradient exponent of quasi-homogeneous polynomials, in particular we generalize the main result of [7] (see also $[4,5,9]$ for related results). Section 5 contains more precise estimates for quasi-homogeneous polynomials of two variables. These results are illustrated by typical examples and completed by a few remarks.

\section{Definitions AND PREliminary Results}

We first recall the concept of a quasi-homogeneous map. Let $\mathbb{K}=\mathbb{C}$ or $\mathbb{R}$. We say that $f: \mathbb{K}^{n} \rightarrow \mathbb{K}^{k}$ is a (positively) quasi-homogeneous map with weight $w:=$ 
$\left(w_{1}, w_{2}, \ldots, w_{n}\right) \in\left(\mathbb{R}_{+}-\{0\}\right)^{n}$ and quasi-degree $d:=\left(d_{1}, d_{2}, \ldots, d_{k}\right) \in\left(\mathbb{R}_{+}-\{0\}\right)^{k}$ if

$$
f_{i}\left(t^{w_{1}} x_{1}, t^{w_{2}} x_{2}, \ldots, t^{w_{n}} x_{n}\right)=t^{d_{i}} f_{i}\left(x_{1}, x_{2}, \ldots, x_{n}\right)
$$

for each $i=1,2, \ldots, k$, and all $t>0$. Note that if $w_{j}=1$ for $j=1,2, \ldots, n$ then the above definition means that the components $f_{i}$ are homogeneous functions of degree $d_{i}$. When $n=1$, a scalar function $f=f_{1}$ satisfying (2.1) is called a (positively) quasihomogeneous function with weight $w=\left(w_{1}, w_{2}, \ldots, w_{n}\right)$ and quasi-degree $d=d_{1}$. In the sequel we shall drop for simplicity the word "positively". Note that any monomial $x^{\alpha}:=x_{1}^{\alpha_{1}} x_{2}^{\alpha_{2}} \ldots x_{n}^{\alpha_{n}}$ is a quasi-homogeneous function with arbitrary weight $w=$ $\left(w_{1}, w_{2}, \ldots, w_{n}\right) \in(\mathbb{N}-\{0\})^{n}$ and quasi-degree $\langle w, \alpha\rangle:=w_{1} \alpha_{1}+w_{2} \alpha_{2}+\cdots+w_{n} \alpha_{n}$. Moreover, we have

Proposition 2.1. Let $f: \mathbb{K}^{n} \rightarrow \mathbb{K}$ be a polynomial function. Then $f$ is quasihomogeneous with weight $w \in(\mathbb{N}-\{0\})^{n}$ and quasi-degree $m \in \mathbb{N}-\{0\}$ if and only if all its constitutive monomials are quasi-homogeneous functions with weight $w$ and quasi-degree $m$.

Proof. Suppose that $f$ is a quasi-homogeneous polynomial with weight $w$ and quasidegree $m$. We have the following finite expansion

$$
f(x):=\sum_{\alpha} a_{\alpha} x^{\alpha}
$$

Then

$$
\sum_{\alpha} a_{\alpha} t^{\langle w, \alpha\rangle} x^{\alpha}=t^{m} \sum_{\alpha} a_{\alpha} x^{\alpha}
$$

This gives

$$
\sum_{\alpha} a_{\alpha}\left[t^{\langle w, \alpha\rangle-m}-1\right] x^{\alpha}=0
$$

for all $x \in \mathbb{K}^{n}$ and for all $t>0$. Since the field $\mathbb{K}$ is of characteristic 0 , we get

$$
\langle w, \alpha\rangle-m=0
$$

for all $\alpha$ provided $a_{\alpha} \neq 0$. In other words, all constitutive monomials of $f$ are quasihomogeneous functions with weight $w$ and quasi-degree $m$. The converse is clear.

For a fixed weight $w:=\left(w_{1}, w_{2}, \ldots, w_{n}\right) \in\left(\mathbb{R}_{+}-\{0\}\right)^{n}$ we set

$$
\|x\|_{w}:=\max _{j=1,2, \ldots, n}\left|x_{j}\right|^{\frac{1}{w_{j}}}
$$

for $x:=\left(x_{1}, x_{2}, \ldots, x_{n}\right) \in \mathbb{K}^{n}$. In the special case $w=w_{0}=(1, \ldots 1)$, we recover the usual $l^{\infty}$ norm on $\mathbb{K}^{n}$ and we set

$$
\|x\|:=\|x\|_{w_{0}}=\max _{j=1,2, \ldots, n}\left|x_{j}\right|
$$


Remark 2.2. (i) It is worth noting that, except in the special case $w=w_{0}=(1, \ldots 1)$, $\|\cdot\|_{w}$ is not a norm since it is not homogeneous of degree 1 .

(ii) It is easy to see that $\|\cdot\|_{w}$ is a quasi-homogeneous function with weight $w$ and quasi-degree 1.

The following basic properties will be used throughout the text.

\section{Proposition 2.3. Let}

$$
\begin{aligned}
w_{*} & :=\min _{j=1,2, \ldots, n} w_{j}, \\
w^{*} & :=\max _{j=1,2, \ldots, n} w_{j} .
\end{aligned}
$$

Then the following hold

(i) For all $\|x\| \geq 1$ we have

$$
\|x\|^{\frac{1}{w_{*}}} \geq\|x\|_{w} \geq\|x\|^{\frac{1}{w^{*}}} .
$$

In particular, $\|x\| \rightarrow \infty$ if and only if $\|x\|_{w} \rightarrow \infty$.

(ii) For all $\|x\| \leq 1$ we have

$$
\|x\|^{\frac{1}{w_{*}}} \leq\|x\|_{w} \leq\|x\|^{\frac{1}{w^{*}}}
$$

In particular, $\|x\| \rightarrow 0$ if and only if $\|x\|_{w} \rightarrow 0$.

Proof. The proof of the proposition is straightforward from the definitions.

In the sequel for $t>0$, for any $w:=\left(w_{1}, w_{2}, \ldots, w_{n}\right) \in\left(\mathbb{R}_{+}-\{0\}\right)^{n}$ and $x:=$ $\left(x_{1}, x_{2}, \ldots, x_{n}\right) \in \mathbb{K}^{n}$ we denote

$$
t \bullet x:=\left(t^{w_{1}} x_{1}, t^{w_{2}} x_{2}, \ldots, t^{w_{n}} x_{n}\right),
$$

and for $d:=\left(d_{1}, d_{2}, \ldots, d_{k}\right) \in\left(\mathbb{R}_{+}-\{0\}\right)^{k}$ we set

$$
\begin{aligned}
d_{*} & :=\min _{i=1,2, \ldots, k} d_{i}, \\
d^{*} & :=\max _{i=1,2, \ldots, k} d_{i} .
\end{aligned}
$$

Let $f: \mathbb{K}^{n} \rightarrow \mathbb{K}^{k}$ be quasi-homogeneous with weight $w:=\left(w_{1}, w_{2}, \ldots, w_{n}\right) \in$ $\left(\mathbb{R}_{+}-\{0\}\right)^{n}$ and quasi-degree $d:=\left(d_{1}, d_{2}, \ldots, d_{k}\right) \in\left(\mathbb{R}_{+}-\{0\}\right)^{k}$. If $f_{i} \equiv 0$ for some $i \in\{1,2, \ldots, k\}$, then $d_{i}$ can be replaced by any positive number. In the sequel we shall assume

$$
\forall i \in\{1,2, \ldots, k\}, \quad f_{i} \not \equiv 0 .
$$

It is easy to check that in this case $d_{i}$ is uniquely defined by (2.1) for all $i \in$ $\{1,2, \ldots, k\}$. Then $d_{*}$ and $d^{*}$ are well defined. 
The next two results summarize some important consequences of the quasi homogeneity property.

Proposition 2.4. Let $f:=\left(f_{1}, f_{2}, \ldots, f_{k}\right): \mathbb{K}^{n} \rightarrow \mathbb{K}^{k}$ be a quasi-homogeneous map with weight $w:=\left(w_{1}, w_{2}, \ldots, w_{n}\right)$ and quasi-degree $d:=\left(d_{1}, d_{2}, \ldots, d_{k}\right)$ satisfying (2.2). Then the following properties are equivalent.

(i) The origin is an isolated zero of $f$.

(ii) $f^{-1}(0)=\{0\}$.

Proof. It is clear that (ii) implies (i). Conversely if (ii) is not satisfied, let $a \neq 0$ be such that $f(a)=0$. This implies that $f_{1}(a)=f_{2}(a)=\cdots=f_{k}(a)=0$. Hence

$$
f_{i}(t \bullet a)=t^{d_{i}} f_{i}(a)=0
$$

for $i=1,2, \ldots, k$ and all $t>0$. Note that $\|t \bullet a\| \rightarrow 0$ as $t \rightarrow 0$. Thus, the origin is not an isolated zero of $f$, which is a contradiction.

Proposition 2.5. Let $f: \mathbb{R}^{n} \rightarrow \mathbb{R}$ be a continuous quasi-homogeneous function with weight $w$ and quasi-degree $m$. Suppose that $n \geq 2$. Then the following conditions are equivalent

(i) $f^{-1}(0)=\{0\}$.

(ii) $f$ has a strict global extremum at the origin.

(iii) For each $\epsilon \geq 0,\left\{x \in \mathbb{R}^{n}|| f(x) \mid=\epsilon\right\}$ is a non-empty compact set.

(iv) $\min _{\|x\|=1}|f(x)|>0$.

Proof. (i) $\Rightarrow$ (ii) If $f^{-1}(0)=\{0\}$, then by connectedness $f$ has a constant sign (for instance $f>0$ ) on the unit Euclidian sphere $\mathbb{S}^{n-1}:=\left\{x \in \mathbb{R}^{n} \mid\|x\|=1\right\}$ of dimension $(n-1)$ if $n>1$. But for any $x \neq 0$, there is clearly $t>0$ such that $y:=t \bullet x$ is in $\mathbb{S}^{n-1}$. Indeed the euclidian norm of $t \bullet x$ is 0 for $t=0$, tends to infinity with $t$ and is a continuous function of $t$, hence it must take the value 1 for some finite positive $t$. Then $f(x)=f\left(t^{-1} \bullet y\right)=t^{-m} f(y)>0$, which proves (ii).

(ii) $\Rightarrow$ (iv) Suppose, by contradiction, that $\min _{\|x\|=1}|f(x)|=0$. Then there exists a point $a \in \mathbb{R}^{n}$ such that $\|a\|=1$ and $f(a)=0$. This implies that $f(t \bullet a)=0$ for all $t>0$, which contradicts (ii).

(iv) $\Rightarrow$ (iii) By contradiction, assume that the set $\left\{x \in \mathbb{R}^{n}|| f(x) \mid=\epsilon\right\}$ is not compact for some $\epsilon \geq 0$. This means that there exists a sequence $x^{p} \in \mathbb{R}^{n}, p \in \mathbb{N}$, such that $\left\|x^{p}\right\| \rightarrow \infty$ as $p \rightarrow \infty$ and $\left|f\left(x^{p}\right)\right|=\epsilon$. Let $t_{p}:=\frac{1}{\left\|x^{p}\right\|_{w}} \rightarrow 0$. Then the sequence $\left|f\left(t_{p} \bullet x^{p}\right)\right|=t_{p}^{m} \epsilon$ tends to zero as $p \rightarrow \infty$. From the sequence of points $t_{p} \bullet x^{p}$ lying on the compact set $\left\{\|x\|_{w}=1\right\}$ one can choose a subsequence convergent to some $a,\|a\|_{w}=1$. Clearly, $f(a)=0$ and $a \neq 0$, which contradicts (iv). 
(iii) $\Rightarrow$ (i) If $f(a)=0$ for some $a \neq 0$, then $f(t \bullet a)=0$ for all $t>0$. Consequently, by letting $t$ tend to infinity, we obtain that the set $\left\{x \in \mathbb{R}^{n}|| f(x) \mid=0\right\}$ is not compact, which contradicts (iii).

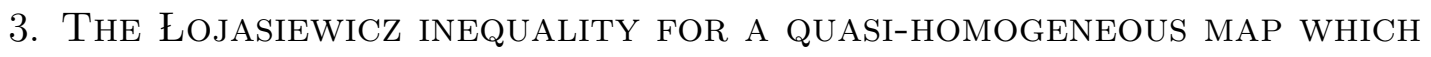 VANISHES ONLY AT THE ORIGIN}

In this section we are interested in the first Łojasiewicz inequality which relates in general the size of $f(u)$ and the distance of $u$ to the set $f^{-1}(0)$. However we essentially restrict our study to the case where this set is reduced to 0 .

Proposition 3.1. Let $f:=\left(f_{1}, f_{2}, \ldots, f_{k}\right): \mathbb{K}^{n} \rightarrow \mathbb{K}^{k}$ be a continuous quasi homogeneous map with weight $w:=\left(w_{1}, w_{2}, \ldots, w_{n}\right)$ and quasi-degree $d:=\left(d_{1}, d_{2}, \ldots, d_{k}\right)$. Then the following statements hold.

(i) There exists a positive constant $c_{1}$ such that

$$
\|f(x)\| \leq c_{1}\|x\|_{w}^{d_{*}}, \quad \text { as } \quad\|x\| \leq 1 .
$$

(ii) If $f^{-1}(0)=\{0\}$, then there exists a positive constant $c_{2}$ such that

$$
c_{2}\|x\|_{w}^{d^{*}} \leq\|f(x)\|, \quad \text { as } \quad\|x\| \leq 1
$$

Proof. Consider the family of topological closed spheres

$$
S_{t}:=\left\{\left(x_{1}, x_{2}, \ldots, x_{n}\right) \in \mathbb{K}^{n} \mid\|x\|_{w}=t\right\} .
$$

Then, by Proposition 2.3(i), for each $t>0$ we have that $S_{t}$ is a compact set. Let $x \in \mathbb{K}^{n}$ be such that $x \neq 0$ and $\|x\| \leq 1$. By Proposition 2.3(ii), we have $t:=\frac{1}{\|x\|_{w}} \geq 1$. Note that $t \bullet x \in S_{1}$.

(i) Let

$$
c_{1}:=\max _{i=1,2, \ldots, k} \max _{y \in S_{1}}\left|f_{i}(y)\right|
$$

We have

$$
c_{1} \geq\left|f_{i}(t \bullet x)\right|=\left|t^{d_{i}} f_{i}(x)\right| \quad \text { for } \quad i=1,2, \ldots, k \text {. }
$$

It follows that

$$
c_{1}\|x\|_{w}^{d_{i}} \geq\left|f_{i}(x)\right| \quad \text { for } \quad i=1,2, \ldots, k .
$$

Consequently,

$$
c_{1}\|x\|_{w}^{d_{*}}=c_{1} \max _{i=1,2, \ldots, k}\|x\|_{w}^{d_{i}} \geq\|f(x)\|
$$

which proves (i). 
(ii) Let $c_{2}:=\min _{y \in S_{1}}\|f(y)\|>0$. By definition, we have

$$
\begin{aligned}
c_{2} & \leq\|f(t \bullet x)\|=\max _{i=1,2, \ldots, k}\left|t^{d_{i}} f_{i}(x)\right| \leq \max _{i=1,2, \ldots, k}\left|t^{d_{i}}\right| \max _{i=1,2, \ldots, k}\left|f_{i}(x)\right| \\
& \leq\left|t^{d^{*}}\right|\|f(x)\|=\frac{1}{\|x\|_{w}^{d^{*}}}\|f(x)\|,
\end{aligned}
$$

which proves (ii). The proposition is proved.

Theorem 3.2. Let $f:=f^{0}+f^{1}+\cdots+f^{l}: \mathbb{K}^{n} \rightarrow \mathbb{K}^{k}$, where $f^{0}, f^{1}, \ldots, f^{l}$ are continuous quasi-homogeneous maps with weight $w=\left(w_{1}, w_{2}, \ldots, w_{n}\right)$ and quasidegrees $d^{0}, d^{1}, \ldots, d^{l}$ respectively such that

$$
\left(d^{0}\right)^{*}<\left(d^{1}\right)_{*} \leq\left(d^{2}\right)_{*} \leq \cdots \leq\left(d^{l}\right)_{*} .
$$

If the origin is an isolated zero of $f^{0}$ then there exists a positive constant $c$ such that

$$
c\|x\|_{w}^{\left(d^{0}\right)^{*}} \leq\|f(x)\|, \quad \text { as } \quad\|x\| \ll 1 .
$$

Proof. By Proposition 3.1(ii), there exists a positive constant $c_{0}$ such that

$$
c_{0}\|x\|_{w}^{\left(d^{0}\right)^{*}} \leq\left\|f^{0}(x)\right\|, \quad \text { as } \quad\|x\| \ll 1 .
$$

On the other hand, from Proposition 3.1(i), there exist positive constants $c_{1}, c_{2}, \ldots, c_{l}$ such that for $i=1,2, \ldots, l$,

$$
\left\|f^{i}(x)\right\| \leq c_{i}\|x\|_{w}^{\left(d^{i}\right)_{*}}, \quad \text { as } \quad\|x\| \ll 1 .
$$

We have for $\|x\| \ll 1$ the next estimate

$$
\begin{aligned}
\left\|f^{1}(x)+f^{2}(x)+\cdots+f^{l}(x)\right\| & \leq\left\|f^{1}(x)\right\|+\left\|f^{2}(x)\right\|+\cdots+\left\|f^{l}(x)\right\| \\
& \leq c_{1}\|x\|_{w}^{\left(d^{1}\right)_{*}}+c_{2}\|x\|_{w}^{\left(d^{2}\right)_{*}}+\cdots+c_{l}\|x\|_{w}^{\left(d^{l}\right)_{*}} .
\end{aligned}
$$

Thus it follows from $\left(d^{0}\right)^{*}<\left(d^{1}\right)_{*} \leq\left(d^{2}\right)_{*} \leq \cdots \leq\left(d^{l}\right)_{*}$ that

$$
\left\|f^{1}(x)+f^{2}(x)+\cdots+f^{l}(x)\right\| \ll\|x\|_{w}^{\left(d^{0}\right)^{*}}, \quad \text { as } \quad\|x\| \ll 1 .
$$

Therefore, we have for $\|x\| \ll 1$ the next inequality

$$
\begin{aligned}
\|f(x)\| & \geq\left\|f^{0}(x)\right\|-\left\|f^{1}(x)+f^{2}(x)+\cdots+f^{l}(x)\right\| \\
& \geq c_{0}\|x\|_{w}^{\left(d^{0}\right)^{*}}-c^{\prime}\|x\|_{w}^{\left(d^{0}\right)^{*}} \quad\left(0<c^{\prime} \ll c_{0}\right) .
\end{aligned}
$$

This gives

$$
\|f(x)\| \geq\left(c_{0}-c^{\prime}\right)\|x\|_{w}^{\left(d^{0}\right)^{*}}, \quad \text { as } \quad\|x\| \ll 1,
$$

which proves the theorem.

The following is a direct consequence from Proposition 3.1 and Theorem 3.2: 
Corollary 3.3. Under the hypothesis of Theorem 3.2, there exists a positive constant c such that

$$
c\|x\|^{\frac{\left(d^{0}\right)^{*}}{w_{*}}} \leq\|f(x)\|, \quad \text { as } \quad\|x\| \ll 1 .
$$

We define the Łojasiewicz exponent $\alpha_{0}(f)$ of the map $f$ at the origin $0 \in \mathbb{K}^{n}$ as the infimum of the set of all real numbers $l>0$ which satisfy the condition: there exists a positive constant $c$ such that

$$
c\|x\|^{l} \leq\|f(x)\|, \quad \text { as } \quad\|x\| \ll 1 .
$$

If the set of all the exponents is empty we put $\alpha_{0}(f):=+\infty$.

Corollary 3.4. Let $f:=\left(f_{1}, f_{2}, \ldots, f_{k}\right): \mathbb{K}^{n} \rightarrow \mathbb{K}^{k}$ be a continuous quasi-homogeneous map with weight $w:=\left(w_{1}, w_{2}, \ldots, w_{n}\right)$ and quasi-degree $d:=\left(d_{1}, d_{2}, \ldots, d_{k}\right)$. Suppose that $f^{-1}(0)=\{0\}$. Then

$$
\frac{d_{*}}{w_{*}} \leq \alpha_{0}(f) \leq \frac{d^{*}}{w_{*}}
$$

Proof. It follows from Corollary 3.3 that

$$
\alpha_{0}(f) \leq \frac{d^{*}}{w_{*}}
$$

In order to prove the left inequality, let $i, j$ be such that $d_{i}=d_{*}$ and $w_{j}=w_{*}$. Take $a \in \mathbb{K}^{n}$ with the property that $a_{j} f_{i}(a) \neq 0$. Then, asymptotically as $t \rightarrow 0$, we have ${ }^{2}$

$$
\begin{aligned}
\|f(t \bullet a)\| & \simeq t^{d_{*}}, \\
\|t \bullet a\| & \simeq t^{w_{*}} .
\end{aligned}
$$

Consequently,

$$
\|f(t \bullet a)\| \simeq\|t \bullet a\|^{\frac{d_{*}}{w_{*}}} .
$$

By the definition of the Eojasiewicz exponent $\alpha_{0}(f)$, we find that

$$
\frac{d_{*}}{w_{*}} \leq \alpha_{0}(f) .
$$

Example 3.5. (i) Let $f:=\left(f_{1}:=x^{2}+y^{4}, f_{2}:=\left(x^{2}-y^{4}\right)^{2}\right): \mathbb{R}^{2} \rightarrow \mathbb{R}^{2}$. It is easy to check that $f$ is a positive quasi-homogeneous map with weight $w:=(2,1)$ and quasi-degree $d:=(4,8)$. Moreover, $\alpha_{0}(f)=4\left(=\frac{d_{*}}{w_{*}}\right)$.

(ii) Let $f:=\left(f_{1}:=x^{2}-y^{4}, f_{2}:=\left(x^{2}+y^{4}\right)^{2}\right): \mathbb{R}^{2} \rightarrow \mathbb{R}^{2}$. Then $f$ is a positive quasihomogeneous map with weight $w:=(2,1)$ and quasi-degree $d:=(4,8)$. Moreover, $\alpha_{0}(f)=8\left(=\frac{d^{*}}{w_{*}}\right)$.

\footnotetext{
${ }^{2}$ Where $A \simeq B$ means that $A / B$ lies between two positive constants.
} 
Corollary 3.6. Let $f: \mathbb{K}^{n} \rightarrow \mathbb{K}$ be a continuous quasi-homogeneous function with weight $w$ and quasi-degree $m$. If $f^{-1}(0)=\{0\}$ then

$$
\alpha_{0}(f)=\frac{m}{w_{*}} .
$$

Proof. The claim comes from $d^{*}=d_{*}=m$.

\section{The ŁoJAsiewicz GRAdient INEQUALITY FOR QUASI-HOMOGENEOUS POLYNOMIALS}

We now consider the case $k=1$ and let $f: \mathbb{K}^{n} \rightarrow \mathbb{K}$ be a $C^{1}$ quasi-homogeneous function with weight $w:=\left(w_{1}, w_{2}, \ldots, w_{n}\right)$ and quasi-degree $m$ :

$$
f\left(t^{w_{1}} x_{1}, t^{w_{2}} x_{2}, \ldots, t^{w_{n}} x_{n}\right)=t^{m} f\left(x_{1}, x_{2}, \ldots, x_{n}\right) .
$$

We define the Eojasiewicz gradient exponent $\beta_{0}(f)$ of the map $f$ at the origin $0 \in \mathbb{K}^{n}$ as the infimum of the set of all real numbers $l>0$ which satisfy the condition: there exists a positive constant $c$ such that

$$
c|f(x)|^{l} \leq\|\nabla f(x)\| \quad \text { for } \quad\|x\| \ll 1 .
$$

If the set of all the exponents is empty we put $\beta_{0}(f):=+\infty$. It is well-known (see [14]) that if $f$ is analytic, then $\beta_{0}(f)<1$.

We start with a general result valid for $C^{1}$ functions.

Theorem 4.1. Let $f: \mathbb{K}^{n} \rightarrow \mathbb{K}$ be a $C^{1}$ quasi-homogeneous function with weight $w$ and quasi-degree $m$. Then

$$
\max \left\{0,1-\frac{w^{*}}{m}\right\} \leq \beta_{0}(f) \leq 1
$$

Proof. Since $f$ is a $C^{1}$-positive quasi-homogeneous function with weight $w$ and quasidegree $m \geq w^{*}$,

$$
m t^{m-1} f(x)=\sum_{j=1}^{n} w_{j} t^{w_{j}-1} x_{j} \frac{\partial f}{\partial x_{j}}(t \bullet x) .
$$

In particular, we have the generalized Euler identity

$$
m f(x)=\sum_{j=1}^{n} w_{j} x_{j} \frac{\partial f}{\partial x_{j}}(x) .
$$

As a consequence, there exists a positive constant $c_{1}$ such that

$$
c_{1}|f(x)| \leq\|x\|\|\nabla f(x)\| \quad \text { for all } \quad\|x\| \ll 1 .
$$

This implies that $\beta_{0}(f) \leq 1$. 
Next, let $l>0$ and $c_{2}>0$ be such that

$$
c_{2}|f(x)|^{l} \leq\|\nabla f(x)\| \text { for all }\|x\| \ll 1 .
$$

We have for all $t>0$ the following relation

$$
t^{w_{j}} \frac{\partial f}{\partial x_{j}}(t \bullet x)=t^{m} \frac{\partial f}{\partial x_{j}}(x) \text { for } \quad j=1,2, \ldots, n .
$$

This shows that

$$
\frac{\partial f}{\partial x_{j}}(t \bullet x)=t^{m-w_{j}} \frac{\partial f}{\partial x_{j}}(x) \text { for } \quad j=1,2, \ldots, n .
$$

Then we have for all $t>0$ the following equations

$$
\begin{aligned}
f(t \bullet x) & =t^{m} f(x), \\
\frac{\partial f}{\partial x_{j}}(t \bullet x) & =t^{m-w_{j}} \frac{\partial f}{\partial x_{j}}(x) \text { for } j=1,2, \ldots, n .
\end{aligned}
$$

Since $f \not \equiv 0$ there is $a \in \mathbb{K}^{n}$ such that $0 \neq \nabla f^{2}(a)=2 f(a) \nabla f(a)$. This implies $f(a) \neq 0$ and $\nabla f(a) \neq 0$. Then, asymptotically as $t \rightarrow+0$, we have

$$
\begin{aligned}
|f(t \bullet a)| & \simeq t^{m}, \\
\|\nabla f(t \bullet a)\| & \simeq t^{m-w_{j}} \quad \text { for some } \quad j \in\{1,2, \ldots, n\},
\end{aligned}
$$

Therefore (4.2) implies the existence of $c_{3}>0$ such that

$$
c_{3} t^{m l} \leq t^{m-w_{j}} \leq t^{m-w^{*}}
$$

as $t \rightarrow+0$. This in turn implies that $m l \geq m-w^{*}$, which is equivalent to

$$
l \geq 1-\frac{w^{*}}{m} .
$$

This, together with the definition of $\beta_{0}(f)$, implies the desired result.

In the special case where 0 is the only critical point of $f$ we have a more precise estimation as follows.

Corollary 4.2. Let $f: \mathbb{K}^{n} \rightarrow \mathbb{K}$ be a $C^{1}$ quasi-homogeneous function with weight $w$ and quasi-degree $m \geq w^{*}$. Suppose that $\nabla f^{-1}(0)=\{0\}$. Then

$$
1-\frac{w^{*}}{m} \leq \beta_{0}(f) \leq 1-\frac{w_{*}}{m} \text {. }
$$

Proof. One has only to show that

$$
\beta_{0}(f) \leq 1-\frac{w_{*}}{m} .
$$

Indeed, by the generalized Euler identity (4.1), there exists a positive constant $c_{1}$ such that

$$
|f(x)| \leq c_{1}\|\nabla f(x)\|\|x\|
$$


On the other hand, it follows from (4.3) that the following

$$
\nabla f(x): \mathbb{K}^{n} \rightarrow \mathbb{K}^{n}, \quad x \mapsto\left(\frac{\partial f}{\partial x_{1}}, \frac{\partial f}{\partial x_{2}}, \ldots, \frac{\partial f}{\partial x_{n}}\right),
$$

is a continuous quasi-homogeneous map with weight $w$ and quasi-degree $\left(m-w_{1}, m-\right.$ $\left.w_{2}, \ldots, m-w_{n}\right)$.

Therefore, by Proposition 2.3 and then Proposition 3.1(ii), for all $\|x\| \ll 1$ we have

$$
\begin{aligned}
|f(x)| & \leq c_{1}\|\nabla f(x)\|\|x\|_{w}^{w_{*}} \\
& \leq c_{2}\|\nabla f(x)\|\|\nabla f(x)\|^{\frac{w_{*}}{m-w_{*}}}=c_{2}\|\nabla f(x)\|^{1+\frac{w_{*}}{m-w_{*}}},
\end{aligned}
$$

for some $c_{2}>0$. Hence there exists a positive constant $c$ such that

$$
c|f(x)|^{1-\frac{w_{*}}{m}} \leq\|\nabla f(x)\| \quad \text { for } \quad\|x\| \ll 1 .
$$

Consequently, by the definition of the Eojasiewicz gradient exponent $\beta_{0}(f)$, we obtain

$$
\beta_{0}(f) \leq 1-\frac{w_{*}}{m},
$$

which completes the proof.

Example 4.3. (i) (see [10]). Let $f: \mathbb{C}^{2} \rightarrow \mathbb{C},(x, y) \mapsto x^{3}+3 x y^{k}, k \geq 3$, be a complex polynomial. It is clear that $f$ is a quasi-homogeneous polynomial with weight $w=(k, 2)$ and quasi-degree $m=3 k$. A direct computation shows that the origin in $\mathbb{C}^{2}$ is an isolated critical point of $f$. Moreover, it follows from the results in [10] and [18] that

$$
\begin{aligned}
\alpha_{0}(\nabla f) & =\frac{3 k}{2}-1 \\
\beta_{0}(f) & =\frac{\alpha_{0}(\nabla f)}{1+\alpha_{0}(\nabla f)}=1-\frac{2}{3 k} .
\end{aligned}
$$

(ii) Let $f: \mathbb{C}^{2} \rightarrow \mathbb{C},(x, y) \mapsto x^{4}-4 x y$, be a complex polynomial. It is clear that $f$ is a quasi-homogeneous polynomial with weight $w=(1,3)$ and quasi-degree $m=4$. A direct computation shows that the origin in $\mathbb{C}^{2}$ is an isolated critical point of $f$. Moreover, it follows from the results in [10] and [18] that

$$
\begin{aligned}
\alpha_{0}(\nabla f) & =1, \\
\beta_{0}(f) & =\frac{\alpha_{0}(\nabla f)}{1+\alpha_{0}(\nabla f)}=\frac{1}{2} .
\end{aligned}
$$

Remark 4.4. Let $f: \mathbb{C}^{n} \rightarrow \mathbb{C}$ be a complex polynomial function with an isolated singularity at 0 . Then from the works of Teissier [18, Corollary 2] we have the following equation

$$
\beta_{0}(f)=\frac{\alpha_{0}(\nabla f)}{1+\alpha_{0}(\nabla f)}
$$


Moreover, Gwoździewicz has remarked, [6], that the above relation fails to hold for some real polynomial functions with an isolated singularity at 0 . However, we have the following.

Corollary 4.5. (see also [6, Theorem 1.3]) Let $f: \mathbb{R}^{n} \rightarrow \mathbb{R}$ be a quasi-homogeneous polynomial function with weight $w$ and quasi-degree $m \geq w^{*}$. If $f^{-1}(0)=\{0\}$, then

$$
\begin{aligned}
\alpha_{0}(f) & =\frac{m}{w_{*}}, \\
\alpha_{0}(\nabla f) & =\alpha_{0}(f)-1=\frac{m}{w_{*}}-1, \\
\beta_{0}(f) & =\frac{\alpha_{0}(\nabla f)}{1+\alpha_{0}(\nabla f)}=1-\frac{w_{*}}{m} .
\end{aligned}
$$

Proof. In fact, by Corollary 3.6, we have

$$
\alpha_{0}(f)=\frac{m}{w_{*}} .
$$

Then the remained relations follow from [6, Theorem 1.3]. We will give below a direct proof in order to keep our paper self-contained.

We first note that the origin is an isolated critical point of $f$. Indeed, if $\nabla f(a)=0$ for some $a \neq 0$, then it follows easily from the generalized Euler identity (4.1) that $f(a)=0$, which is a contradiction.

Without loss of generality, we may suppose that $f(x) \geq 0$ for all $x \in \mathbb{R}^{n}$ with equality if and only if $x=0$. For each $\delta>0$, the restriction of $f$ to the sphere $\{x \in$ $\left.\mathbb{R}^{n} \mid\|x\|_{E}=\delta\right\}$ attains its minimum at least one point, where $\|x\|_{E}:=\sqrt{\sum_{i=1}^{n} x_{i}^{2}}$. Let

$$
\Gamma:=\left\{u \in \mathbb{R}^{n} \mid f(u)=\min _{\|x\|_{E}=\|u\|_{E}} f(x)\right\} .
$$

It follows from the Tarski-Seidenberg theorem (see, for example, [3, Theorem 2.3.4]), that $\Gamma$ is semi-algebraic. Hence the Curve Selection Lemma [15] is applicable. Together with Lagrange's Multipliers Theorem, this implies that there exists an analytic $\operatorname{map}(\lambda, \varphi):(-\epsilon, \epsilon) \rightarrow \mathbb{R} \times \mathbb{R}^{n}, \tau \mapsto(\lambda(\tau), \varphi(\tau))$, such that

(i) $\varphi(\tau)=0$ if and only if $\tau=0$;

(ii) $\varphi(\tau) \in \Gamma$ for all $\tau \in[0, \epsilon)$; and

(iii) $\nabla f(\varphi(\tau))=\lambda(\tau) \varphi(\tau)$ for all $\tau \in[0, \epsilon)$.

Let $a t^{p}, a>0$, be the leading term of the Taylor expansion of $\|\varphi(\tau)\|^{2}$, and $b t^{q}, b \neq 0$, be that of $|f(\varphi(\tau))|^{2}$. Then, asymptotically as $t \rightarrow 0$, we have

$$
|f(\varphi(\tau))| \simeq\|\varphi(\tau)\|^{\frac{q}{p}}
$$

Consequently, by the definition of $\alpha_{0}(f)$, we get

$$
\alpha_{0}(f) \geq \frac{q}{p}
$$


On the other hand, we may assume (taking $\epsilon>0$ small enough if necessary) that the function $\tau \mapsto\|\varphi(\tau)\|$ is strictly increasing. Together with the condition (i), we find that for each $x \in \mathbb{R}^{n},\|x\| \ll 1$, there exists a positive number $\tau \in[0, \epsilon)$ satisfying the relation $\|\varphi(\tau)\|_{E}=\|x\|_{E}$. Hence,

$$
|f(x)|=f(x) \geq \min _{\|u\|_{E}=\|x\|_{E}} f(u)=f(\varphi(\tau)) \simeq\|\varphi(\tau)\|^{\frac{q}{p}}=\|x\|^{\frac{q}{p}} .
$$

By the definition, thus

$$
\alpha_{0}(f) \leq \frac{q}{p}
$$

Therefore,

$$
\alpha_{0}(f)=\frac{q}{p} .
$$

Moreover, it follows from the generalized Euler identity (4.1) that

$$
m f(\varphi(\tau))=\sum_{j=1}^{n} w_{j} \varphi_{j}(\tau) \frac{\partial f}{\partial x_{j}}(\varphi(\tau)) .
$$

By the condition (iv), hence

$$
\begin{aligned}
|m f(\varphi(\tau))| & =|\lambda(\tau)| \sum_{j=1}^{n} w_{j}\left[\varphi_{j}(\tau)\right]^{2} \\
& =\frac{\|\nabla f(\varphi(\tau))\|}{\|\varphi(\tau)\|} \sum_{j=1}^{n} w_{j}\left[\varphi_{j}(\tau)\right]^{2} \\
& \simeq\|\varphi(\tau)\|\|\nabla f(\varphi(\tau))\| .
\end{aligned}
$$

In particular, we get

$$
\|\nabla f(\varphi(\tau))\| \simeq\|\varphi(\tau)\|^{\frac{q}{p}-1} \simeq|f(\varphi(\tau))|^{1-\frac{p}{q}}
$$

By definitions, hence

$$
\begin{aligned}
\alpha_{0}(\nabla f) & \geq \frac{q}{p}-1=\alpha_{0}(f)-1=\frac{m}{w_{*}}-1, \\
\beta_{0}(f) & \geq 1-\frac{p}{q}=1-\frac{1}{\alpha_{0}(f)}=1-\frac{w_{*}}{m} .
\end{aligned}
$$

Then the corollary follows immediately from Corollaries 3.4 and 4.2.

The following result is of general interest but we shall only use it to prove Theorem 4.7 below.

Lemma 4.6. Let $f: \mathbb{K}^{n} \rightarrow \mathbb{K}$ be a $C^{1}$-function. For each $k$ positive integer, consider the function $\tilde{f}: \mathbb{K}^{n} \rightarrow \mathbb{K}, x \mapsto[f(x)]^{k}$. Suppose that there exist $c>0$ and $\theta \in(0,1]$ such that

$$
c|\widetilde{f}(x)|^{1-\theta} \leq\|\nabla \widetilde{f}(x)\| \quad \text { for } \quad\|x\| \ll 1 .
$$


Then

$$
\frac{c}{k}|f(x)|^{1-k \theta} \leq\|\nabla f(x)\| \quad \text { for } \quad\|x\| \ll 1 .
$$

Proof. We have

$$
\nabla \widetilde{f}(x)=k[f(x)]^{k-1} \nabla f(x)
$$

Hence

$$
c\left|[f(x)]^{k}\right|^{1-\theta} \leq k\left|[f(x)]^{k-1}\right|\|\nabla f(x)\| .
$$

This implies

$$
\frac{c}{k}|f(x)|^{1-k \theta} \leq\|\nabla f(x)\|,
$$

concluding the proof of the lemma.

The following is a generalization of [7, Theorem 2.1].

Theorem 4.7. Let $f: \mathbb{R}^{2} \rightarrow \mathbb{R}$ be a quasi-homogeneous polynomial function with weight $w:=\left(w_{1}, w_{2}\right)$ and quasi-degree $m$. Then there exists a positive constant $c$ such that

$$
c|f(x, y)|^{1-\frac{w_{*}}{m}} \leq\|\nabla f(x, y)\|, \quad \text { as } \quad\|(x, y)\| \ll 1 .
$$

Proof. Without loss of generality, we may suppose that

$$
1 \leq w_{*}=w_{1} \leq w_{2} .
$$

There are two cases to be considered.

Case 1. $m$ is divisible by $w_{1}$; i.e., $q:=\frac{m}{w_{1}}$ is a positive integer number.

Consider the following function

$$
g(x, y):=f\left(x, y^{\frac{w_{2}}{w_{1}}}\right)
$$

Then, by Proposition 2.1, we can see that $g$ is a homogeneous polynomial on $\mathbb{R} \times \mathbb{R}_{+}$ of degree $q=\frac{m}{w_{1}}$. Indeed we can write for some finite set $S \subset \mathbb{N} \times \mathbb{N}$ :

$$
f(x, y)=\sum_{\alpha:=\left(\alpha_{1}, \alpha_{2}\right) \in S} a_{\alpha} x^{\alpha_{1}} y^{\alpha_{2}}
$$

with

$$
w_{1} \alpha_{1}+w_{2} \alpha_{2}=m=q w_{1} .
$$

Hence

and therefore

$$
\alpha_{2}=\left(q-\alpha_{1}\right) \frac{w_{1}}{w_{2}}
$$

$$
f(x, y)=\sum_{\alpha \in S} a_{\alpha} x^{\alpha_{1}} y^{\left(q-\alpha_{1}\right) \frac{w_{1}}{w_{2}}}
$$


which provides

$$
g(x, y)=\sum_{\alpha \in S} a_{\alpha} x^{\alpha_{1}} y^{q-\alpha_{1}}
$$

It now follows from [7, Theorem 2.1] that there exists a positive constant $c$ such that

$$
c|g(x, y)|^{1-\frac{w_{1}}{m}} \leq\|\nabla g(x, y)\|, \quad \text { as } \quad\|(x, y)\| \ll 1 \text { and } y \geq 0 .
$$

On the other hand, by the definition

$$
\begin{aligned}
& \frac{\partial g}{\partial x}(x, y)=\frac{\partial f}{\partial x}\left(x, y^{\frac{w_{2}}{w_{1}}}\right), \\
& \frac{\partial g}{\partial y}(x, y)=\frac{w_{2}}{w_{1}} y^{\frac{w_{2}}{w_{1}}-1} \frac{\partial f}{\partial y}\left(x, y^{\frac{w_{2}}{w_{1}}}\right) .
\end{aligned}
$$

Therefore, asymptotically as $(x, y) \rightarrow(0,0)$ and $y \geq 0$,

$$
\begin{aligned}
c\left|f\left(x, y^{\frac{w_{2}}{w_{1}}}\right)\right|^{1-\frac{w_{1}}{m}} & \leq\left\|\left(\frac{\partial f}{\partial x}\left(x, y^{\frac{w_{2}}{w_{1}}}\right), \frac{w_{2}}{w_{1}} y^{\frac{w_{2}}{w_{1}}-1} \frac{\partial f}{\partial y}\left(x, y^{\frac{w_{2}}{w_{1}}}\right)\right)\right\| . \\
& \leq\left\|\left(\frac{\partial f}{\partial x}\left(x, y^{\frac{w_{2}}{w_{1}}}\right), \frac{\partial f}{\partial y}\left(x, y^{\frac{w_{2}}{w_{1}}}\right)\right)\right\|,
\end{aligned}
$$

because $w_{2} \geq w_{1}$.

Let $u:=y^{\frac{w_{2}}{w_{1}}} \geq 0$. Then

$$
c|f(x, u)|^{1-\frac{w_{1}}{m}} \leq\left\|\left(\frac{\partial f}{\partial x}(x, u), \frac{\partial f}{\partial y}(x, u)\right)\right\| .
$$

By an entirely analogous argument but replacing $g(x, y)=f\left(x, y^{\frac{w_{2}}{w_{1}}}\right)$ by $f\left(x,-y^{\frac{w_{2}}{w_{1}}}\right)$ we can show that the above inequality also holds for all $u \leq 0$. These prove the theorem in Case 1.

Case 2. $m$ is not divisible by $w_{1}$.

Let $\widetilde{f}(x, y):=[f(x, y)]^{w_{1}}$. Then it is clear that $\widetilde{f}(x, y)$ is a positive quasi-homogeneous polynomial with weight $\left(w_{1}, w_{2}\right)$ and quasi-degree $\widetilde{m}:=m w_{1}$. Since $\frac{\widetilde{m}}{w_{1}}=m$ is an integer number, by applying Case 1 for the polynomial $\tilde{f}$ we get

$$
\widetilde{c}|\tilde{f}(x, y)|^{1-\frac{w_{1}}{\tilde{m}}} \leq\|\nabla \tilde{f}(x, y)\|, \quad \text { as } \quad\|(x, y)\| \ll 1,
$$

for some $\widetilde{c}>0$.

By Lemma 4.6, we get

$$
\frac{\widetilde{c}}{w_{1}}|f(x, y)|^{1-\frac{w_{1}}{m}} \leq\|\nabla f(x, y)\|
$$

which completes the proof of the theorem.

Remark 4.8. As we see in the next proposition, the result of Theorem 4.7 is no longer valid in dimensions $n>2$. 
Proposition 4.9. (Compare with [7, Remark 2.4]) Let $f: \mathbb{R}^{3} \rightarrow \mathbb{R}$ be given by

$$
f(x, y, z):=x^{4}+x^{2} z^{2}-2 x y^{2} z+y^{4}=x^{4}+\left(x z-y^{2}\right)^{2} .
$$

Then there exists a curve $\varphi:[0, \epsilon) \rightarrow \mathbb{R}^{n}, t \mapsto \varphi(t)$, such that

$$
\|\nabla f[\varphi(t)]\| \ll|f[\varphi(t)]|^{1-\frac{1}{4}} \quad \text { for } \quad 0<t \ll 1 .
$$

In particular, $\beta_{0}(f)>1-\frac{w_{*}}{m}=1-\frac{1}{4}$.

Proof. It is clear that $f$ is a weighted quasi-homogeneous polynomial with weight $w=(1,1,1)$ and quasi-degree $m=4$. Moreover, $f$ has non-isolated zero at the origin; namely, $f^{-1}(0)=\{(0,0, t) \mid t \in \mathbb{R}\}$. Define the polynomial curve $\varphi:[0, \epsilon) \rightarrow \mathbb{R}^{3}, t \mapsto$ $(x(t), y(t), z(t))$, by

$$
\begin{aligned}
x(t) & :=t^{2}, \\
y(t) & :=t+t^{5}, \\
z(t) & :=1 .
\end{aligned}
$$

One easily verifies that

$$
\begin{aligned}
f[\varphi(t)] & =t^{8}+4 t^{12}+4 t^{16}+t^{20}, \\
\frac{\partial f}{\partial x}[\varphi(t)] & =-2 t^{10}, \\
\frac{\partial f}{\partial y}[\varphi(t)] & =8 t^{7}+12 t^{11}+4 t^{15}, \\
\frac{\partial f}{\partial z}[\varphi(t)] & =-4 t^{8}-2 t^{12} .
\end{aligned}
$$

Hence, asymptotically as $t \rightarrow 0$,

$$
\|\nabla f[\varphi(t)]\| \simeq t^{7} \ll t^{6} \simeq|f[\varphi(t)]|^{1-\frac{1}{4}} .
$$

This completes the proof.

Remark 4.10. The polynomial $x^{4}+x^{2} z^{2}-2 x y^{2} z+y^{4}$ in the above proposition is a homogenization of $x^{4}+\left(x-y^{2}\right)^{2}$ by the new variable $z$. The last one is a polynomial in the class of polynomials which was considered by János Kollár ([8]).

The following is a direct consequence of Theorem 4.1 and Theorem 4.7.

Corollary 4.11. Let $f: \mathbb{R}^{2} \rightarrow \mathbb{R}$ be a quasi-homogeneous polynomial function with weight $w:=\left(w_{1}, w_{2}\right)$ and quasi-degree $m$. Then

$$
\max \left\{0,1-\frac{w^{*}}{m}\right\} \leq \beta_{0}(f) \leq 1-\frac{w_{*}}{m} .
$$




\section{Additional Results, REmarks And eXAmples in Dimension 2}

In this section we will denote by $f: \mathbb{R}^{2} \rightarrow \mathbb{R}$ a quasi-homogeneous polynomial with weight $w=\left(w_{1}, w_{2}\right)$ and quasi-degree $m$ such that

$$
w_{*}=w_{1} \leq w_{2}=w^{*} \leq m .
$$

We now apply Corollary 4.11 in special cases.

Corollary 5.1. If the origin is an isolated zero of $f_{y}$ then

$$
\beta_{0}(f)=1-\frac{w_{2}}{m} \text {. }
$$

Proof. It is well known that $f_{y}$ is quasi-homogeneous polynomial with weight $w=$ $\left(w_{1}, w_{2}\right)$ and quasi-degree $m-w_{2}$. Since $f_{y}^{-1}(0)=\{0\}$, it follows from Proposition 2.5 that the polynomial $f_{y}$ has a strict global extremum at the origin. Thus we can assume that $f_{y}>0$ on $\mathbb{R}^{n}-\{0\}$. By again Proposition 2.5, the set $\left\{f_{y}=1\right\}$ is nonempty compact. Hence

$$
\infty>c:=\max _{f_{y}(u, v)=1}|f(u, v)|>0 .
$$

Take any $(x, y) \in \mathbb{R}^{2}$. Let $\epsilon:=\left[f_{y}(x, y)\right]^{\frac{1}{m-w_{2}}}$. Then $\left\{(u, v) \in \mathbb{R}^{2} \mid f_{y}(u, v)=\epsilon^{m-w_{2}}\right\}$ is a non empty compact set. Moreover,

$$
\begin{aligned}
|f(x, y)| \leq \max _{f_{y}(u, v)=\epsilon^{m-w_{2}}}|f(u, v)| & =\max _{f_{y}\left(\epsilon^{-w_{1}} u, \epsilon^{\left.-w_{2} v\right)=1}\right.}|f(u, v)| \\
& =\max _{f_{y}(\tilde{u}, \tilde{v})=1}\left|f\left(\epsilon^{w_{1}} \tilde{u}, \epsilon^{w_{2}} \tilde{v}\right)\right| \\
& =\max _{f_{y}(\tilde{u}, \tilde{v})=1}|f(\tilde{u}, \tilde{v})| \epsilon^{m} \\
& =c\left[f_{y}(x, y)\right]^{\frac{m}{m-w_{2}}} .
\end{aligned}
$$

This gives

$$
f_{y}(x, y) \geq c^{\prime}|f(x, y)|^{1-\frac{w_{2}}{m}}
$$

here $c^{\prime}:=c^{1-\frac{w_{2}}{m}}>0$. Therefore

$$
\|\nabla f(x, y)\| \geq\left|f_{y}(x, y)\right| \geq c^{\prime}|f(x, y)|^{1-\frac{w_{2}}{m}} .
$$

By the definition of $\beta_{0}(f)$, we get

$$
\beta_{0}(f) \leq 1-\frac{w_{2}}{m}
$$

Then, by Corollary 4.11, $\beta_{0}(f)=1-\frac{w_{2}}{m}$. 
Example 5.2. Let $f: \mathbb{R}^{2} \rightarrow \mathbb{R},(x, y) \mapsto y^{3}+3 x^{4} y+2 x^{6}$ be a real polynomial. It is clear that $f$ is a quasi-homogeneous polynomial with weight $w=(1,2)$ and quasi-degree $m=6$. A direct computation shows that $f_{y}^{-1}(0)=\{(0,0)\}$. Hence $\beta_{0}(f)=1-\frac{2}{6}=\frac{2}{3}$.

Corollary 5.3. Suppose that the origin is not an isolated zero of $f_{y}$. If there exists $(a, b) \in \mathbb{R}^{2}$ such that $f(a, b) \neq 0, f_{x}(a, b) \neq 0$ and $f_{y}(a, b)=0$, then

$$
\beta_{0}(f)=1-\frac{w_{1}}{m} \text {. }
$$

Proof. By the hypothesis, we have for all $t>0$

$$
\begin{aligned}
f\left(t^{w_{1}} a, t^{w_{2}} b\right) & \simeq t^{m} \\
f_{x}\left(t^{w_{1}} a, t^{w_{2}} b\right) & \simeq t^{m-w_{1}} \\
f_{y}\left(t^{w_{1}} a, t^{w_{2}} b\right) & \equiv 0 .
\end{aligned}
$$

Asymptotically as $t \rightarrow 0$, hence

$$
\begin{aligned}
\left|f\left(t^{w_{1}} a, t^{w_{2}} b\right)\right| & \simeq t^{m} \\
\left\|\nabla f\left(t^{w_{1}} a, t^{w_{2}} b\right)\right\| & \simeq t^{m-w_{1}} .
\end{aligned}
$$

This implies that

$$
\left\|\nabla f\left(t^{w_{1}} a, t^{w_{2}} b\right)\right\| \simeq\left|f\left(t^{w_{1}} a, t^{w_{2}} b\right)\right|^{1-\frac{w_{1}}{m}} .
$$

Then, by the definition of $\beta_{0}(f)$,

$$
\beta_{0}(f) \geq 1-\frac{w_{1}}{m}
$$

On the other hand, by Corollary $4.11, \beta_{0}(f) \leq 1-\frac{w_{1}}{m}$. Therefore $\beta_{0}(f)=1-\frac{w_{1}}{m}$.

Example 5.4. Let $f: \mathbb{R}^{2} \rightarrow \mathbb{R},(x, y) \mapsto x^{2} y-y^{2}$ be a real polynomial. It is clear that $f$ is a quasi-homogeneous polynomial with weight $w=(1,2)$ and quasi-degree $m=4$. A direct computation shows that $f_{y}^{-1}(0)=\left\{x^{2}-2 y=0\right\}$ and the origin in $\mathbb{R}^{2}$ is an isolated critical point of $f$. Moreover, it is easy to see that the conditions of Corollary 5.3 are satisfied. Hence, $\beta_{0}(f)=1-\frac{1}{4}=\frac{3}{4}$.

Remark 5.5. (i) All results in this paper allow to compute the Łojasiewicz exponents for some functions which are not quasi-homogeneous, for instance, the function $f(x):=P(A x)$, where $P$ is a quasi-homogeneous polynomial of two variables and $A$ a nonsingular $2 \times 2$ square matrix. As an example the polynomial $P(x, y):=$ $a x^{4}+b y^{2}+c x^{2} y$ is quasi-homogeneous with weight $(1,2)$ and quasi-degree 4 . The polynomial $Q(x, y)=P(x, x+y)$ is not quasi-homogeneous if $b c \neq 0$. 
(ii) On the other hand, there are of course polynomials of two variables which cannot be put in the form $=P(A x)$ with, $P, A$ as above. For instance the polynomial $Q(x, y):=x^{2}(1+y)$ is such that no polynomial $P=Q \circ A$ with $A$ a nonsingular $2 \times 2$ square matrix is quasi-homogeneous.

\section{REFERENCES}

[1] V. I. Arnold, A. N. Varchenko, and S. M. Gusein-Zade, Singularities of differentiable maps, Birkhauser, 1985.

[2] D. D'Acunto, K. Kurdyka, Explicit bounds for the Eojasiewicz exponent in the gradient inequality for polynomials, Ann. Pol. Math., Vol. 87 (2005), 51-61.

[3] Benedetti R. and J. J. Risler, Real algebraic and semi-algebraic sets, Hermann, 1990.

[4] C. Bivia-Ausina, and S. Encinas, The Eojasiewicz exponent of a set of weighted homogeneous ideals, J. Pure Appl. Algebra 215 (2011), no. 4, 578-88.

[5] S. Brzostowski, T. Krasinski, and G. Oleksik, A conjecture on the Eojasiewicz exponent, J. Singul. 6 (2012), 124-130.

[6] J. Gwoździewicz, The Eojasiewicz exponent of an analytic function at an isolated zero, Comment. Math. Helv. Vol. 74 (1999), No. 3, 364-375.

[7] A. Haraux, Positively homogeneous functions and the Eojasiewicz gradient inequality, Ann. Polon. Math. Vol. 87 (2005), 165-174.

[8] J. Kollar, An effective Eojasiewicz inequality for real polynomials, Period. Math. Hungar., Vol. 38 (1999), N. 3, 213-221.

[9] T. Krasinski, G. Oleksik, and A. Ploski, The Eojasiewicz exponent of an isolated weighted homogeneous surface singularity, Proc. Amer. Math. Soc. 137 (2009), no. 10, 3387-3397.

[10] T. C. Kuo and Y. C. Lu, On analytic function germs of two complex variables, Topology, Vol. 16 (1977), 299-310.

[11] K. Kurdyka, and S. Spodzieja, Separation of real algebraic sets and the Lojasiewicz exponent, Proc. Amer. Math. Soc., 142 (9) S 0002-993912061-2, (2014), 3089-3102.

[12] C. Li, B. S. Mordukhovich, and T. S. Phạm, New fractional error bounds for polynomial systems with applications to Höderian stability in optimization and spectral theory of tensors, Math. Program. Ser. A, DOI: 10.1007/s10107-014-0806-9.

[13] S. Łojasiewicz, Une proprriété topologique des sous ensembles analytiques réels, Colloques internationaux du C.N.R.S, 117. Les Équations aux Dérivées Partielles, (1963) 87-89.

[14] S. Łojasiewicz, Ensembles semi-analytiques, preprint IHES, 1965.

[15] J. Milnor, Singular points of complex hypersurfaces, Annals of Mathematics Studies 61, Princeton University Press, 1968.

[16] T. S. Pham, The Eojasiewicz exponent of a subanalytic mapping at an isolated zero, Proceedings of the American Mathematical Society, 139 (2011), 1-9.

[17] T. S. Phạm, An explicit bound for the Eojasiewicz exponent of real polynomials, Kodai Mathematical Journal, No. 2, 35 (2012), 311-319.

[18] B. Teissier, Varietés polaires I - Invariants polaires des singularités d'hypersufaces, Inventiones Mathematicae, Vol. 40 (1977), 267-293. 